\title{
ELABORACIÓN DEL TEST DE HABILIDADES PARA LA GESTIÓN EN LA NEGOCIACIÓN DE CONFLICTOS
}

\author{
Luis Vicuña P., ${ }^{1}$ Héctor Hernández V., Mildred Paredes T.y José Rios D. \\ Universidad Nacional Mayor de San Marcos, Lima, Perú \\ (RECIBIDO EL 31/10/2008, ACEPTADO EL 16/12/2008)
}

\begin{abstract}
RESUMEN
El presente informe reporta a la comunidad científica el producto de la elaboración del test de habilidades para la gestión en la negociación de conflictos, cuyo objetivo es determinar las habilidades para gestionar éxitosamente la gestión de conflictos. Se parte que este es el resultado de la compleja interacción de cinco habilidades: comunicación, compromiso, perspectiva, control emocional, y empatía; en base a estos supuestos factores se construyó el test, el que fue puesto a consideración de jueces y análisis estadísticos del comportamiento de sus componentes dentro del todo, habiendo encontrado que el test mide lo que se propone desde el punto de vista de los jueces y que los factores son partes que relacionan significativamente con el todo, a su vez para conocer el error de medición se utilizó el método del test retest y el método de la consistencia interna de Cronbach, por ambos métodos el coeficiente hallado supera al 0,80 .
\end{abstract}

De lo hallado se concluye que los factores propuestos subyacen en la habilidad de gestión en la negociación del conflictos y el test mide dentro del margen de error permitido.

Palabras clave: Habilidad, gestión, negociación, conflicto.

\begin{abstract}
The present report reports to the scientific community the product of the elaboration of the test of abilities for the management in the negotiation of conflicts, whose objective is to determine the abilities to manage the management of conflicts successful; part that this is the result of the complex interaction of five abilities: communication, commitment, perspective, emotional control, and empathy; on the basis of these supposed factors the test was constructed, the one that was put to consideration of judges and statistic analyses of the behavior of its components within the whole, having found that the test measures what it sets out from the point of view of the judges and who the factors are parts that they significantly relate to the whole, his you see to know the error measurement was used the method of the test retest and the method of the internal consistency of Cronbach, by both methods the found coefficient surpasses to the 0,80 .
\end{abstract}

1 Docente Principal de la Facultad de Psicología de la UNMSM. E-mail: uiisvicunaperi@hotmail.com 
Of the found thing one concludes that the proposed factors proposed they underlie in the ability of management in the negotiation of the conflicts and the test measures within the allowed allowable error.

Keywords: Ability, management, negotiation, conflict.

\section{INTRODUCCIÓN}

Sin lugar a mucha discusión, vivimos en una época en la que casi todo se negocia. La negociación se encuentra, sin discriminacion de los grupos sociales, como una forma de relación permanente. Las naciones, los gobiernos, las empresas, los gremios y las personas, utilizan gran parte de su energía en procesos de negociación. La persona se enfrenta también a negociaciones cotidianas, tales como cuando va a adquirir una vivienda, o tal vez un carro, o ponerse de acuerdo para pasar un fin de semana.

La convivencia dentro de la sociedad enfrenta los deseos y necesidades de las personas; lo que favorece a uno parece perjudicar a otro, este hecho afecta nuestra "imagen pública" y lo que es más importante a la relación entre las partes, que deben cuidarse mediante estrategias comunicativas para resolver los conflictos y avanzar hacia acuerdos satisfacctorios y lo más duraderos posible.

Sin lugar a duda, las habilidades de negociación implican el uso apropiado de técnicas de comunicación pues se elaboran estrategias, tácticas y estilos de negociación, así mismo cuando se prepara la discusión, la comunicación no verbal como herramientas para disminuir la agresión y facilitar las relaciones, atenuando en lo posible las consecuencias negativas cuando el conflicto no ha sido resuelto eficazmente.

Desde este punto de vista, nuestro objetivo consiste en elaborar un test que permita medir las habilidades para la gestión en la negociación de conflictos. Para lo cual nos proponemos despejar las interrogantes:

¿En qué medida los componentes de comunicación, compromiso, control emocional, perspectiva, y empatía son habilidades para la gestion en la negociación de conflictos?

¿El test, a través de los componentes para medir habilidades para la gestión en la negociación de conflictos, cumple su propósito con el menor error de medición permitido?

De lo planteado se desprende que nuestro principal resultado será identificar los componentes de la comunicación que tienen mayor incidencia en la gestión cuando se negocia conflictos. Las unidades participantes son profesionales vinculados a la administración del conocimiento, específicamente directores de centros educativos del nivel primario y secundario; asimismo, el test que pretendemos elaborar permitirá detectar las habilidades de las personas que cumplen funciones directivas en la gestión de la negociación de conflictos y comprobar la confiabilidad de los indicadores para medir con el menor error posible las habilidades para gestionar la negociación de conflictos; y proporcionar a la comunidad científica normas que permitan obtener el perfil diagnóstico de las personas que asuman cargos como mediadores de conflictos o también como directivos de organizaciones de diferentes índoles. 
La importancia del presente estudio está asociada a las confrontaciones humanas inevitales en el proceso de socialización; la habilidad para enfrentarlas y resolverlas saludablemente es posible en la medida que se consiga interdependencia positiva de las metas (Fauvet, 1975, en Munduate, L. y Martinez, J. M, 1998). Para Deutsch (1973), los conflictos actúan como el motor transformador de la sociedad y el impulso íntimo de superación. Observamos que los conflictos, desde estos puntos de vista, son impulsores, por ello no debemos evitarlos sino optimizar las habilidades que conducen a la eficacia de las medidas pertinentes de carácter transformador, para lo cual es necesario tener herramientas válidas y confiables que permitan identificar y predecir el éxito de la gestión durante la negociación, de esta manera se podrá evitar fracasos que acarrean consecuencias negativas no sólo para las personas involucradas sino para su entorno.

El test tendrá como componentes: los tipos y estilos así como los elementos de la negociación (Malaret, 2001); otro componente será el lenguaje y las técnicas de comunicación, que tiene que ver con los tipos de comunicación (Rodríguez, 1993), tanto la comunicación verbal como la no verbal, ambos aplicados en situación de conflicto donde la habilidad de escuchar activamente, el cálculo en la discusión, la cooperación, la identificación de señales y barreras, son de vital importancia dentro del enfoque sociocultural, de actuación de un individuo dentro de una colectividad (Jenney y Arendt, 1992).

La negociación es un medio alternativo de resolución de conflictos entre las personas y entre las organizaciones e instituciones, sin la presencia de terceros, en la cual las partes en conflicto exponen y defienden sus puntos de vista. Constituye un proceso de comunicación entre las partes a través del cual ellas mismas dan solución al conflicto llegando a un mutuo acuerdo que privilegie sus intereses.

La negociación, según Fischer, R. y Ury, W. (1981), también es una comunicación de un lado al otro y viceversa, que se utiliza para llegar a un acuerdo cuando las partes tienen algunos intereses compartidos y otros controvertidos. Para Gulliver, P. (1979), la negociación es un proceso de intercambio de información que propicia el mutuo aprendizaje y el constante ajuste de expectativas y preferencias de las partes. Mientras que Shea, G. (1983) califica a la negociación creativa como un proceso en el que dos o más personas se reúnen y, a través de discusiones en las que interviene la imaginación y la creatividad, se enfrentan a un problema y llegan a una solución innovadora, la que mejor satisface a las necesidades e intereses de las partes, y consigue el compromiso de todos en el cumplimiento del acuerdo.

Sea cual sea la temática implícita en una negociación, ésta es siempre un proceso de interacción y comunicación entre personas que defienden unos intereses determinados que se perciben como incompatibles, en búsqueda de armonizar criterios, donde se neutraliza el sentimiento de perdedor.

El conflicto es el estado de indecisión en que vive la persona ante situaciones que le generan mínimamente dos conductas de aproximación ante dos situaciones diferentes, con la peculiaridad que sólo una de ellas puede emitir. Otras veces el conflicto define la situación donde queremos evitar dos conductas, pero necesariamente tendremos que optar por una.

Hay situaciones más complicadas aún donde la misma situación genera dos conductas contradictorias. 
Los conflictos nos conducen a penosos estados, penosos porque implican reacciones emocionales ambivalentes, donde, incluso cuando nos llevan al agrado, sobreviene el sufrimiento causado por la privación o la inhibición de otro de los componentes, con sus consecuentes estados emocionales; estos estados con tales peculiaridades podemos identificarlos en los siguientes tipos estudiados experimentalmente por Hovland y Miller, en Kimble. G, (1969):

Aproximación-Aproximación, ejemplo el mismo día programan dos espectáculos de nuestro agrado en el mismo horario, como es lógico tendremos que optar por uno de ellos.

Evitación-Evitación, en este conflicto hay dos opciones, las dos son "feas" para nosotros, pero tenemos que optar por una; por ejemplo en casos necesarios cuando o se toma un purgante o le ponen una enema, o se queda con hambre o come el platillo que no le agrada.

Aproximación-Evitación, hay situaciones donde, la complejidad del hecho nos pone en situación crítica para elegir porque es la misma situación, hecho o estímulo el que no genera dos reacciones diferentes; por ejemplo a una persona le agrada la playa por el sol, el atractivo del paisaje, pero le tiene terror al agua fría, o también me agrada aprobar el curso, pero me fastidia estudiar, o en las organizaciones me agradan mis derechos pero no cumplir con mis deberes.

¿Se aprenden los conflictos? Sí. ¿Podemos aprender a ser conflictivos? Sí. podemos distinguir que en los conflictos hay dos componentes, el primero es el subyacente a las conductas que son de carácter natural y corresponden a las emociones, y el segundo componente son los disparadores o los iniciadores de la conducta conflictiva, que en el común de los casos son aprendidos, por ejemplo el cine o el teatro en un inicio son neutrales no generan reacción emocional, pero la experiencia con ellos, se habrán conectado a otros estímulos que sí producían emociones placenteras, por ejemplo, las golosinas o la compañía, o el espectáculo en sí mismo, que desencadenaron reacciones emocionales que fueron adornando de "color" y de "temperatura" a tales acontecimientos. Modelo parecido puede extenderse a los alimentos, a las personas, a las acciones, a los roles y funciones, a los fenómenos a los comportamientos organizacionales y hacia el mismo recurso humaño.

En la medida que aprendamos que el conflicto nos puede generar ganancias secundarias, corremos el riesgo de utilizarlo como herramienta; por ejemplo, cuando decimos de alguien "No le hagamos renegar que le puede subir la presión y morirse", ¿qué está ocurriendo?, pues aquella persona, con sus reacciones autonómicas ante estados de cólera, ha descubierto que nos puede manipular con sus somatizaciones, o también cuando las personas que nos gratifican de diferentes formas utilizan la situación conflictiva para que se inviertan los roles, y seamos ahora nosotros quienes lo gratificamos.

En sí el conflicto es para el hombre otra herramienta de control de la conducta, que se utiliza individualmente en las relaciones personales y que las personas la van orquestando basándose en las aproximaciones o en las evitaciones que más o menos se encuentran distribuidas en el grupo o entre los miembros de una organización, para hacerlo notorio y formar alianzas en los causales del conflicto compartido, para hacer fuerza o presión a fin de obtener beneficios y estos no son más que atenuar bajo la esperanza que estamos en camino de la solución definitiva. 
Los conflictivos saben bien que jamás se resolverán al cien por ciento las causales, saben además que el hombre requiere sentirse bien, pero como este es un estado transitorio, es fácil encontrar una nueva causa de conflicto, alimentarla y volver a mantener viva la esperanza de resolverlo siempre con el apoyo de todos.

Este proceso organizacional descrito funciona también en lo íntimo, en lo personal, cuando es uno mismo quien busca un motivo para sentirse mal y hacer sentir mal, para buscar luego la forma de solucionarlo y encontrar la tranquilidad y el consuelo de ser un efectivo negociador, sin reparar que fue él mismo quien generó, con la complicidad de una persona de su entorno, que hasta podría ser muy querido. Al parecer vivir en armonía, en paz, en apoyo solidario, es un deseo posible de alcanzar; sin embargo, es el mismo individuo quien renuncia a ello cada momento de su vida.

Desde esta perspectiva, no podemos esperar que no existan conflictos, estos están; por ello será buena práctica desarrollar el hábito de pensar cuánto de nuestra cuota hemos puesto en el presente conflicto, y cuando lo hayamos resuelto, cuánto tiempo pasaremos hasta alimentar o construir uno nuevo.

Como no podemos evitar que ocurran los conflictos, hagamos algo interesante con ellos, desarrollemos habilidades que nos sirvan para crecer, esto ocurrirá cuando la solución de un conflicto resulte un producto, sobre el cual generaremos una nueva necesidad, hacia un nuevo producto, buscando que cada causa sea sustentable y que los productos resulten sostenibles, esto es una vida adulta y no neurótica, donde echamos la culpa a los demás, al sistema y a todo lo genérico, en estos casos la búsqueda de la solución suele ser fenomenológica, existencial, que actúa como un consuelo para continuar soportando la vida que nos tocó vivir y negándonos la posibilidad de asumir el rol adulto en el manejo del conflicto.

La habilidad es un componente de la inteligencia (Cronbach, L., 1972; Guilford, 1986) que se refiere a la capacidad o la aptitud potencial para ejecutar una tarea, una destreza que aún no ha sido aprendida; implica las habilidades figurativas, simbólicas, semántica y conductual, que las personas son capaces de aprender en cuanto existe la base biológica que permite procesar, dentro de un periodo madurativo, la solución correcta de problemas de estos tipos, a partir de los cuales ocurre la organización de nuevos conocimientos expresados en lo que podemos llamar conductas manifiestas y exitosas, que en el presente estudio nos servirán para la negociación de conflictos.

Las habilidades consideradas en el presente estudio son: de comunicación, de compromiso, de control emocional, de perspectiva, de empatía. Estas habilidades implican fundamentalmente el uso eficaz de herramientas de interacción donde la comunicación verbal y no verbal es el canal que permite la decodificación y encodificación que demandan de habilidades figurativas, simbólicas semánticas y conductuales, que se expresan en cómo se escucha, cómo se responde gestual y posturalmente, cómo se responde verbalmente, situación que ocasiona la reacción verbal o no verbal.

Otra habilidad tiene que ver con la capacidad de la persona para involucrarse con el conflicto, en el sentido de sentirse cómodo participando en su solución, se expresa en la identificación con el papel de negociador lejos del compromiso por normatividad o por necesidad. 
La habilidad de perspectiva, en el sentido de J. Piaget (1969): como la representación mental, por la cual la persona es capaz de reconstruir mentalmente un percepto desde la óptica de un tercero o del otro, se expresa cuando la persona es capaz de interpretar un acontecimiento no linealmente sino a partir del dato observable llegar a la interpretación total, como a partir de un plaño percibimos un sólido.

La habilidad de control emocional se refiere al uso de recursos personales o potenciales para evitar que la carga emocional que llega al negociador en el lenguaje verbal y no verbal, en el compromiso normativo irracional y en el compromiso de necesidad, así como la anticipación interpretativa a partir de un hecho o indicador, termine por bloquear emocionalmente, irritando o asumiendo posturas preferenciales, o estresándolo, disminuyendo la eficiencia y eficacia negociadora.

La empatía es otra de las habilidades que se presenta mediante la capacidad del negociador para abandonar sus puntos de vista y asumir en cada instante los puntos de vista de la persona o las personas en conflicto.

Si las personas tienen las habilidades descritas y aplicadas a la negociación estará en condiciones de:

Descubrir cuándo los conflictos "están a punto" o si ya maduraron lo suficiente como para someterse a un proceso de mediación, es decir se estará en condiciones de configurar los siguientes requisitos:

- Cuando los conflictos son extremadamente complejos y prolongados en el tiempo.

- Cuando los esfuerzos por controlar o administrar el conflicto bilateralmente, es decir, por las propias partes contendientes, han llegado a un "impasse".

- Cuando ninguno de los oponentes está dispuesto a seguir tolerando los costos crecientes de una escalada del conflicto.

Comprender la necesidad de elaborar el mapa del conflicto, puesto que negociar supone aplicar un conjunto de técnicas que parten del sentido común y del cultivo de habilidades para acercar a las partes y reflejar sus necesidades y preocupaciones. Los negociadores y los posibles mediadores necesitan un mapa de las vías conceptuales, o "mapa del conflicto" implícito en la discrepancia, en el que deben detallar al menos lo siguiente:

- $\quad$ los motivos que han dado pie al conflicto (las causas normalmente son varias),

- los problemas de relación entre las partes,

- las discrepancias en la interpretación de los hechos,

- $\quad$ los intereses incompatibles,

- las barreras estructurales,

- $\quad$ las diferencias de valores,

- $\quad$ los obstáculos que se oponen al arreglo los procedimientos destinados a encauzar o resolver la disputa,

- $\quad$ los factores individuales o estructurales que podrían mejorar la relación los puntos de coincidencia y los valores comunes. 
Un buen "mapa del conflicto" permitirá después elaborar una buena agenda de negociaciones. El tema del conflicto, diagnóstico y alternativas de afrontamiento, son prioritarios en el trabajo profesional del psicólogo en todas sus áreas de aplicación, incluyendo la investigación básica.

La habilidad para la gestión implica el uso de recursos verbales y no verbales para realizar las acciones pertinentes en dirección a las metas; la eficiencia y eficacia dependerán de la conjunción de las herramientas seleccionadas y utilizadas en la elaboración estratégica del abordale y afrontamiento.

Conocer los componentes que optimizan la gestión de la negociación de conflictos permitirá, por sus consecuencias, mejorar el sentimiento de autovalía personal y reconocimiento social, como efectivo solucionador de problemas.

En nuestro país donde la diversidad cultural es una de las barreras para lograr metas comunes este tipo de estudio tiene un efecto positivo hacia la búsqueda o conciliación de metas.

El presente estudio permitirá reducir, adenás, las improvisaciones mediante la detección de los pasos y caracteristicas funcionales que debemos tener:

- En el abordaje de la conciliación que podrá reducir tiempo y costos en las confrontaciones.

- En ambientes laborales, podra conducir en el menor tiempo a la solución de conflictos.

- En ambientes académicos, los malos entendidos y la emergencia de conflictos por desgaste o estrés podrán prevenirse o afrontarlos con solvencia.

- En la familia, los terapeutas familiares tendrán una herramienta que les permita evaluar cómo gestionar la negociación de los conflictos para el abordaje con el menor costo por quiebre emocional.

De lo descrito se desprende que los conflictos son situaciones donde necesariamente habrá un punto de vista o decisión a tomar en contra de otro, generando estados antagónicos de aproximación-aproximación, evitación-evitación y aproximaciones y evitaciones múltiples, donde la habilidad del negociador consiste en destacar lo valioso de los dos puntos de vista o situaciones, pero que, a la luz de las circunstancias, de los recursos y del momento actual, sólo es posible asumir un punto de vista o situación y que este proceso sea asimilado con tolerancia, capaz de contrarrestar reacciones emocionales. Este es el papel de la comunicación en símbolo y signo, conseguir que las personas arriben a un desenlace que resulte conveniente y productivo bajo las circunstancias actuales; es decir, el negociador debe poseer habilidades verbales y no verbales para arribar con éxito a la solución del conflicto. De ser así, tales recursos adquiridos y desarrollados por el mediador conducirán a cualquier situación crítica, a convergencias favorables donde las habilidades verbales y no verbales para colocar información tienen un rol fundamental, de esta manera estamos en condiciones de sustentar la siguiente hipótesis:

H1. Las técnicas de comunicación verbales y no verbales relacionan significativamente con los indicadores de habilidad para la gestión de la negociación del conflicto. 


\section{MÉTODO}

La presente investigación es de tipo aplicada, con método correlacional comparativo, diseño transversal (Hernández, R. y otros 2003).

La muestra fue aleatoria representativa al 0.05 con un nivel de confianza del 0.95 habiendo elegido a 115 instituciones educativas de las cuales sólo 105 directores colaboraron con el presente estudio.

La primera fase consistió en los procedimientos de validación; empezamos por la validez de contenido de la tabla de consistencia mediante el criterio de expertos, luego se elaboró el test piloto el cual fue aplicado para la validación semántica de los ítemes. Habiendo efectuado las correcciones y ajustes pertinentes, se elaboró la prueba definitiva, la misma que fue aplicada a la muestra. Con los resultados se averiguó el error de medida mediante los métodos de confiabilidad: consistencia interna y el test retest.

Los ítemes fueron analizados por el poder discriminativo utilizando el coeficiente biserial y tetracórico.

La validez del cuestionario se determinó mediante el método de constructo orientado al conocimiento de la variabilidad y la estructura de los componentes para lo cual se correlacionó cada subtest con la puntuación total y con cada uno de ellos. Finalmente, con los resultados depurados, se elaboró la norma interpretativa que facilita la elaboración del pérfil diagnóstico de la habilidad para la gestión en la negociación del conflicto.

\section{RESULTADOS}

\section{Validez de contenido}

La tabla de especificaciones con las operacionalizaciones, indicadores y los ítemes fueron consultados con expertos en negociación de conflictos, psicólogos consultores, consejeros familiares, religiosos católicos, abogados en la parte civil y algunos dirigentes sindicales, quienes debían opinar sobre tres puntos específicos: Si las cinco dimensiones consideradas son válidas desde el punto de vista de su experiencia; si las operacionalizaciones son pertinentes; y si los ítemes hacen referencia a las dos primeras. Fueron diez expertos en total y sus respuestas se validaron con el análisis del Ji cuadrado (véase resultados en la tabla N. $\left.{ }^{o} 1\right)$.

\section{Análisis de ítemes}

Para verificar en qué medida los ítemes discriminan los criterios de respuesta a partir de los examinados, que en más del $80 \%$ declaran poseer el atributo respecto al $20 \%$ que se declara contrario, y habiendo calculado los valores promedios de las respuestas escalares y contrastado mediante la razón crítica de diferencia medias, se ha encontrado los valores que se muestran en la tabla N. ${ }^{\circ}$, además hemos calculado mediante el coeficiente biserial la correlación ítem-test total como doble criterio para discriminar y validar las unidades de medición del test en estudio. 
Finalmente, hemos verificado si las medidas superficiales expresadas por los totales son, en alguna medida, expresión de factores no medidos directamente, y que las inferimos a través de los coeficientes de correlación encontrados, que en el presente estudio son los cinco factores que correlacionan significativamente con el puntaje total, dejando entrever que la puntuación total obtenida guarda relación con los factores que las subyacen, tal como puede verse en la tabla N. ${ }^{0} 3$. 


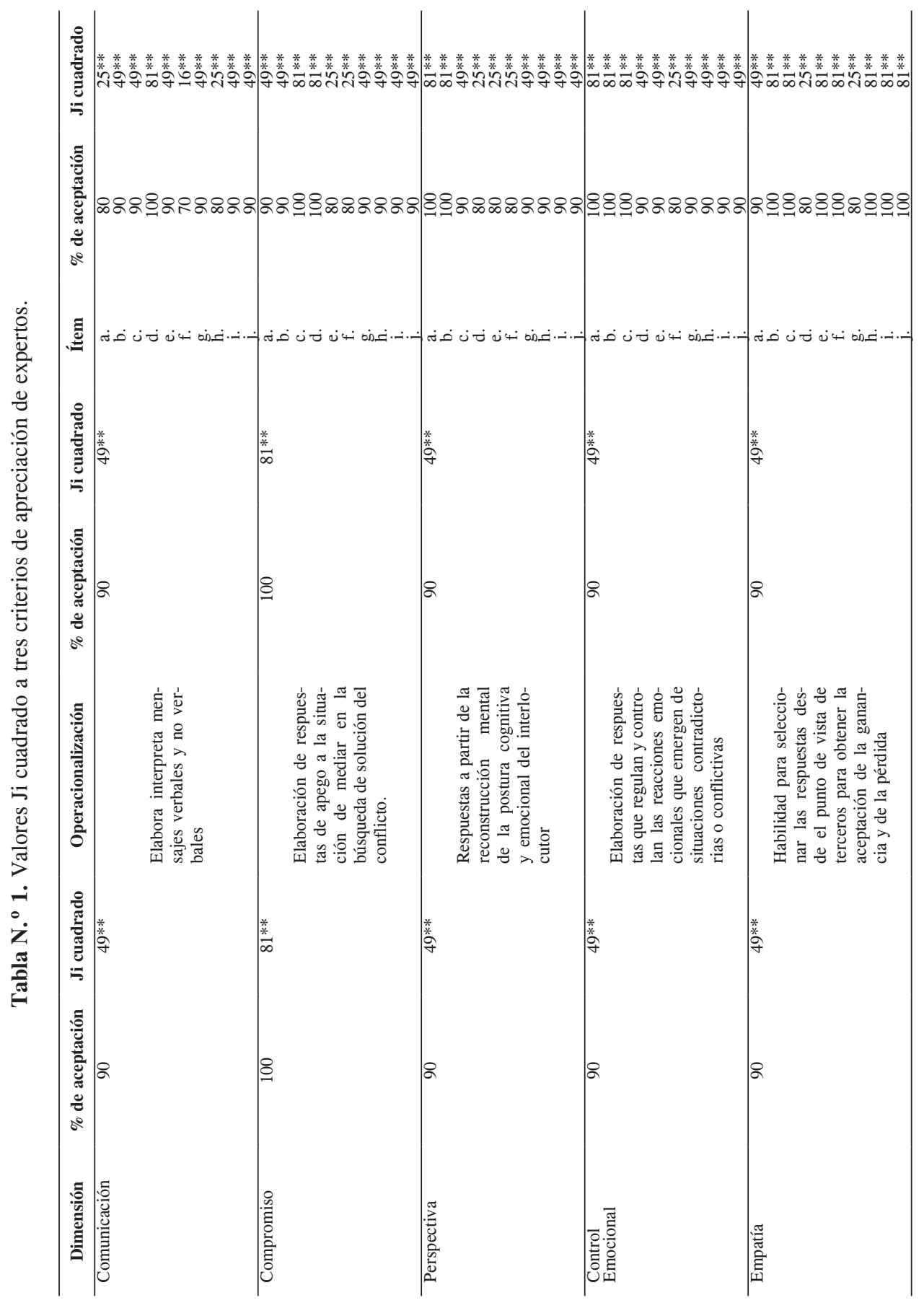


Tabla N. ${ }^{\circ}$ 2. Razón crítica de diferencia de medias por cada ítem y correlaciones biseriales ítem test total del test de habilidades para la gestión en la negociación del conflicto.

\begin{tabular}{|c|c|c|c|c|c|c|c|c|c|}
\hline Ítemes & $\begin{array}{c}\text { Media } \\
\text { Alto }\end{array}$ & $\begin{array}{c}\text { Varianza } \\
\text { Alto }\end{array}$ & $\underset{\text { Media }}{\text { Bajo }}$ & $\begin{array}{c}\text { Varianza } \\
\text { Bajo }\end{array}$ & Diferencia & EE. & $\mathbf{t}$ & $\begin{array}{l}\text { r ítem- } \\
\text { test total }\end{array}$ & Ítemes \\
\hline 2 & 5 & 0,43 & 4,05 & 0,62 & 1 & 0,22 & 4,49 & $0,40 *$ & 2 \\
\hline 7 & 4,9 & 1,23 & 3,9 & 1,42 & 1 & 0,36 & 2,82 & $0,31 *$ & 7 \\
\hline 11 & 4,9 & 0,75 & 3,95 & 1,47 & 0,95 & 0,33 & 2,92 & $0,30 *$ & 11 \\
\hline 16 & 5,5 & 0,44 & 4,29 & 0,78 & 1,19 & 0,24 & 4,95 & $0,50 *$ & 16 \\
\hline 22 & 5,4 & 0,53 & 4,19 & 0,54 & 1,24 & 0,23 & 5,5 & $0,38 *$ & 22 \\
\hline 25 & 4 & 1,66 & 2,81 & 1,2 & 1,24 & 0,37 & 3,35 & $0,41 *$ & 25 \\
\hline 29 & 5 & 0,81 & 3,43 & 1,58 & 1,62 & 0,34 & 4,8 & $0,56^{*}$ & 29 \\
\hline 33 & 3,4 & 2,34 & 2,57 & 1,48 & 0,86 & 0,43 & 2,01 & $0,29 *$ & 33 \\
\hline 39 & 2,4 & 1,19 & 2,38 & 0,9 & 0 & 0,32 & 0 & 0,04 & 39 \\
\hline 45 & 3,2 & 1,8 & 2,67 & 1,46 & 0,57 & 0,39 & 1,45 & $0,20 *$ & 45 \\
\hline 1 & 5 & 1,43 & 4,57 & 1,2 & 0,43 & 0,35 & 1,21 & 0,16 & 1 \\
\hline 4 & 2,7 & 0,98 & 2,57 & 1,86 & 0,1 & 0,37 & 0,26 & 0,11 & 4 \\
\hline 9 & 2,8 & 2,56 & 2,67 & 1,75 & 0,1 & 0,45 & 0,21 & 0,004 & 9 \\
\hline 14 & 4 & 2,19 & 3,14 & 1,65 & 0,86 & 0,43 & 2,01 & $0,29 * *$ & 14 \\
\hline 23 & 4 & 1 & 3,57 & 1,58 & 0,48 & 0,35 & 1,36 & $0,22 *$ & 23 \\
\hline 31 & 5,2 & 0,44 & 4,14 & 0,98 & 1,05 & 0,26 & 4,03 & $0,34 * *$ & 31 \\
\hline 35 & 4,7 & 1,17 & 3,9 & 0,75 & 0,76 & 0,3 & 2,51 & $0,33 * *$ & 35 \\
\hline 38 & 4 & 1,66 & 2,86 & 1,36 & 1,1 & 0,38 & 2,89 & $0,33 * *$ & 38 \\
\hline 41 & 5 & 0,57 & 3,57 & 1,48 & 1,43 & 0,31 & 4,57 & $0,37 * *$ & 41 \\
\hline 46 & 3 & 2 & 2,14 & 0,98 & 0,86 & 0,38 & 2,28 & 0,18 & 46 \\
\hline 3 & 5 & 0,71 & 3,76 & 1,32 & 1,29 & 0,31 & 4,13 & $0,37 * *$ & 3 \\
\hline 6 & 5,1 & 0,6 & 3,86 & 0,88 & 1,29 & 0,27 & 4,84 & $0,38 * *$ & 6 \\
\hline 10 & 5 & 1,19 & 3 & 1,52 & 2,05 & 0,36 & 5,7 & $0,53 * *$ & 10 \\
\hline 15 & 5,4 & 0,33 & 4,24 & 0,56 & 1,14 & 0,21 & 5,54 & $0,38^{* *}$ & 15 \\
\hline 19 & 5,7 & 0,3 & 3,9 & 2,37 & 1,81 & 0,36 & 5,07 & $0,52 * *$ & 19 \\
\hline 27 & 5,2 & 1,3 & 3,62 & 1,57 & 1,57 & 0,37 & 4,25 & $0,42 * *$ & 27 \\
\hline 34 & 3,4 & 2,52 & 2,86 & 1,27 & 0,52 & 0,42 & 1,23 & 0,16 & 34 \\
\hline 42 & 5,3 & 0,98 & 3,62 & 2,24 & 1,71 & 0,39 & 4,38 & $0,37 * *$ & 42 \\
\hline 47 & 5,3 & 0,39 & 3,38 & 1,38 & 1,9 & 0,29 & 6,55 & $0,54 * *$ & 47 \\
\hline 50 & 5 & 0,43 & 3,76 & 0,66 & 1,29 & 0,23 & 5,66 & $0,51 * *$ & 50 \\
\hline 8 & 4,7 & 1,37 & 2,95 & 1,19 & 1,71 & 0,35 & 4,92 & $0,53 * *$ & 8 \\
\hline 13 & 5,1 & 0,66 & 2,9 & 1,8 & 2,19 & 0,34 & 6,4 & $0,63 * *$ & 13 \\
\hline 17 & 4,3 & 1,56 & 2,71 & 1,54 & 1,62 & 0,38 & 4,22 & $0,48 * *$ & 17 \\
\hline 21 & 3,7 & 2,51 & 2,9 & 0,85 & 0,76 & 0,4 & 1,91 & $0,25^{*}$ & 21 \\
\hline 26 & 4,6 & 1,2 & 3,24 & 1,13 & 1,33 & 0,33 & 4 & $0,38^{* *}$ & 26 \\
\hline 30 & 3 & 2,29 & 3,19 & 1,39 & $-0,2$ & 0,42 & $-0,46$ & $-0,05$ & 30 \\
\hline 36 & 4,9 & 1,32 & 3,81 & 0,63 & 1,1 & 0,31 & 3,59 & $0,39 * *$ & 36 \\
\hline 40 & 5,3 & 0,78 & 3,05 & 1,57 & 2,24 & 0,33 & 6,7 & $0,61 * *$ & 40 \\
\hline 44 & 4,9 & 0,56 & 3,57 & 1,2 & 1,33 & 0,29 & 4,61 & $0,56^{* *}$ & 44 \\
\hline 48 & 5,1 & 0,37 & 3,33 & 1,17 & 1,76 & 0,27 & 6,49 & $0,50 * *$ & 48 \\
\hline 5 & 4,9 & 0,66 & 3,76 & 1,32 & 1,14 & 0,31 & 3,72 & $0,42 * *$ & 5 \\
\hline 12 & 5,4 & 0,43 & 3,9 & 1,13 & 1,48 & 0,27 & 5,42 & $0,49 * *$ & 12 \\
\hline 18 & 5,1 & 0,66 & 3,71 & 0,97 & 1,38 & 0,28 & 4,97 & $0,49 * *$ & 18 \\
\hline 20 & 5 & 0,81 & 3,43 & 1,58 & 1,52 & 0,34 & 4,52 & $0,47 * *$ & 20 \\
\hline 24 & 5,3 & 0,59 & 4,43 & 0,91 & 0,86 & 0,27 & 3,21 & $0,23 *$ & 24 \\
\hline 28 & 5,2 & 0,66 & 3,1 & 1,51 & 2,14 & 0,32 & 6,66 & $0,49 * *$ & 28 \\
\hline 32 & 3,1 & 1,71 & 2,29 & 0,87 & 0,81 & 0,35 & 2,31 & $0,28 * *$ & 32 \\
\hline 37 & 4,5 & 1,39 & 2,81 & 1,2 & 1,67 & 0,35 & 4,74 & $0,48^{* *}$ & 37 \\
\hline 43 & 4,1 & 2,03 & 3 & 1,43 & 1,14 & 0,41 & 2,82 & $0,28 * *$ & 43 \\
\hline 49 & 2,9 & 2,37 & 2,76 & 1,9 & 0,14 & 0,45 & 0,32 & 0,04 & 49 \\
\hline
\end{tabular}


Tabla N. ${ }^{\circ}$ 3. Correlaciones intersubtest y test total del test de habilidades para la gestión en la negociación del conflicto.

\begin{tabular}{lcccccc}
\hline \multicolumn{1}{c}{ Habilidades } & Comunicación & Compromiso & Perspectiva & $\begin{array}{c}\text { Control } \\
\text { Emocional }\end{array}$ & Empatía & $\begin{array}{c}\text { Test } \\
\text { Total }\end{array}$ \\
\hline Comunicación & 1 & & & & & \\
Compromiso & $0,58^{*}$ & 1 & & & & \\
Perspectiva & $0,50^{*}$ & $0,42^{*}$ & 1 & & & \\
Control Emocional & $0,62^{*}$ & $0,62^{*}$ & $0,41^{*}$ & 1 & & \\
Empatía & $0,54^{*}$ & $0,57^{*}$ & $0,43^{*}$ & $0,63^{*}$ & 1 & \\
Test Total & $0,83^{* *}$ & $0,80^{* *}$ & $0,69^{* *}$ & $0,84^{* *}$ & $0,80^{* *}$ & 1 \\
\hline
\end{tabular}

$\left({ }^{*}\right) P<0,001$

$\left({ }^{* *}\right) P<0,0001$

\section{Confiabilidad}

La verificación del nivel de error de medición fue mediante el análisis de consistencia interna ítem, test total, además del coeficiente de Cronbach y el coeficiente del test retest, encontrando: Por la consistencia interna e ítem test total, los coeficientes hallados son significativos dejando entrever que los ítems agrupados en cinco factores miden dentro de los errores permitidos, tal como puede verse en la tabla N. ${ }^{\circ} 4$. 
Tabla N. ${ }^{\circ}$ 4. Coeficiente de confiabilidad por consistencia interna con la fórmula de Cronbach.

\begin{tabular}{|c|c|c|c|c|c|}
\hline Ítemes & $\begin{array}{c}\text { Media si } \\
\text { borramos ítem }\end{array}$ & $\begin{array}{c}\text { Varianza si } \\
\text { borramos ítem }\end{array}$ & $r$ ítem - Total & r Múltiple & $\begin{array}{l}\text { Confiabilidad si } \\
\text { borramos ítem }\end{array}$ \\
\hline $\mathrm{V} 2$ & 203,82 & 378,52 & 0,39 & 0,59 & 0,83 \\
\hline V7 & 204,18 & 372,59 & 0,48 & 0,75 & 0,83 \\
\hline V11 & 203,75 & 385,88 & 0,23 & 0,59 & 0,84 \\
\hline V16 & 203,86 & 381,37 & 0,35 & 0,75 & 0,83 \\
\hline V22 & 203,92 & 373,92 & 0,58 & 0,75 & 0,83 \\
\hline V25 & 203,22 & 383,88 & 0,28 & 0,77 & 0,83 \\
\hline V29 & 203,80 & 370,43 & 0,60 & 0,82 & 0,83 \\
\hline V33 & 204,55 & 401,71 & $-0,11$ & 0,67 & 0,84 \\
\hline V39 & 204,00 & 379,17 & 0,45 & 0,74 & 0,83 \\
\hline V45 & 203,32 & 379,47 & 0,45 & 0,66 & 0,83 \\
\hline $\mathrm{V} 1$ & 204,07 & 382,70 & 0,35 & 0,75 & 0,83 \\
\hline V4 & 203,50 & 382,23 & 0,43 & 0,71 & 0,83 \\
\hline V9 & 204,07 & 381,74 & 0,29 & 0,56 & 0,83 \\
\hline V14 & 204,67 & 396,17 & $-0,01$ & 0,53 & 0,84 \\
\hline V23 & 204,15 & 375,82 & 0,38 & 0,69 & 0,83 \\
\hline V31 & 204,95 & 410,24 & $-0,28$ & 0,59 & 0,85 \\
\hline V35 & 204,34 & 383,29 & 0,28 & 0,74 & 0,83 \\
\hline V38 & 204,18 & 370,40 & 0,52 & 0,79 & 0,83 \\
\hline V41 & 203,94 & 387,13 & 0,24 & 0,67 & 0,83 \\
\hline V46 & 204,26 & 377,71 & 0,35 & 0,74 & 0,83 \\
\hline V3 & 203,69 & 381,28 & 0,41 & 0,72 & 0,83 \\
\hline V6 & 204,97 & 383,24 & 0,25 & 0,63 & 0,83 \\
\hline V10 & 204,32 & 381,78 & 0,28 & 0,69 & 0,83 \\
\hline V15 & 204,25 & 383,11 & 0,29 & 0,58 & 0,83 \\
\hline V19 & 204,08 & 389,40 & 0,14 & 0,65 & 0,84 \\
\hline V27 & 205,06 & 395,00 & 0,02 & 0,69 & 0,84 \\
\hline V34 & 204,70 & 388,02 & 0,16 & 0,66 & 0,84 \\
\hline V42 & 203,60 & 379,65 & 0,45 & 0,75 & 0,83 \\
\hline V47 & 204,08 & 395,55 & 0,01 & 0,63 & 0,84 \\
\hline V50 & 204,23 & 386,25 & 0,18 & 0,68 & 0,84 \\
\hline V8 & 204,41 & 392,47 & 0,06 & 0,60 & 0,84 \\
\hline V13 & 203,90 & 380,88 & 0,30 & 0,50 & 0,83 \\
\hline V17 & 204,31 & 391,64 & 0,10 & 0,64 & 0,84 \\
\hline V21 & 203,90 & 383,32 & 0,30 & 0,64 & 0,83 \\
\hline V26 & 203,84 & 388,46 & 0,16 & 0,70 & 0,84 \\
\hline V30 & 203,90 & 372,96 & 0,72 & 0,79 & 0,83 \\
\hline V36 & 204,49 & 375,91 & 0,39 & 0,51 & 0,83 \\
\hline V40 & 204,11 & 369,39 & 0,65 & 0,76 & 0,83 \\
\hline V44 & 204,01 & 374,78 & 0,54 & 0,66 & 0,83 \\
\hline V48 & 204,72 & 387,36 & 0,17 & 0,63 & 0,84 \\
\hline V5 & 203,59 & 379,42 & 0,45 & 0,68 & 0,83 \\
\hline V12 & 203,80 & 385,12 & 0,25 & 0,67 & 0,83 \\
\hline V18 & 204,44 & 387,58 & 0,18 & 0,73 & 0,84 \\
\hline V20 & 203,96 & 388,98 & 0,15 & 0,50 & 0,84 \\
\hline V24 & 203,63 & 379,60 & 0,50 & 0,65 & 0,83 \\
\hline V28 & 203,85 & 379,59 & 0,28 & 0,49 & 0,83 \\
\hline V32 & 204,46 & 376,00 & 0,41 & 0,80 & 0,83 \\
\hline V37 & 204,09 & 378,44 & 0,44 & 0,70 & 0,83 \\
\hline V43 & 203,86 & 378,51 & 0,48 & 0,67 & 0,83 \\
\hline V49 & 204,73 & 399,70 & $-0,07$ & 0,59 & 0,84 \\
\hline
\end{tabular}

Coeficiente de confiabilidad por consistencia interna utilizando la fórmula de Cronbach para un total de 50 ítemes.

Confiabilidad total $=0,8368$ Confiabilidad corregida $=0,8548$ 
Por el método del test retest, con un intervalo de dos meses entre la primera y segunda aplicación con un grupos de sesenta directores de Instituciones Educativas hemos obtenido los siguientes coeficientes:

Tabla N. ${ }^{\circ}$ 5. Coeficiente de confiabilidad por el método del test retest, con intervalo de dos meses en un grupo de sesenta directores cien directores de instituciones educativas en el periodo lectivo 2006.

\begin{tabular}{lc}
\hline \multicolumn{1}{c}{ Habilidades } & r11 \\
\hline Comunicación & $0,86^{* *}$ \\
Compromiso & $0,81^{* *}$ \\
Perspectiva & $0,84^{* *}$ \\
Control Emocional & $0,80^{* *}$ \\
Empatía & $0,86^{* *}$ \\
Test total & $0,91^{* *}$ \\
\hline
\end{tabular}

$(* *) \mathrm{P}<0,0001$

\section{DISCUSIÓN}

Partimos en el presente estudio que las habilidades para la gestión en negociación del conflicto se expresan por la interacción de un conjunto de factores que se sintetizan en la adecuada gestión del conflicto, es decir, el éxito para conseguir que se logre la claridad, se consiga la mayor transparencia y, dentro de los intereses contrapuestos, emerja la solución donde las partes, pese a reconocer que no han conseguido el $100 \%$ de sus expectativas, sin embargo, han logrado la satisfacción de haber encontrado soluciones conciliatorias a partir de las cuales podrán continuar su desarrollo personal, grupal u organizacional.

Las habilidades inherentes a este proceso son, en primer lugar, la comunicación, el compromiso, la perspectiva, el control emocional y la empatía; estos cinco componentes tienen la siguiente base para gestionar adecuadamente el conflicto. El mediador debe tener la capacidad para encodificar sus ideas y decodificar la información de sus oponentes desde el punto de vista de estos, haciendo uso de la empatía para no contaminar los mensajes de los oponentes con sus propias interpretaciones que pueden llevarlo a sesgar la información; esta tarea implica tiempo y muchas veces el mediador se involucra en asuntos que no le incumben directamente a él ni al negociador y sin embargo asume esta responsabilidad por el alto compromiso con esta labor, es decir que sus afectos sean positivos, que se sienta importante ante tal labor, y que al mismo tiempo esté convencido que perdería mucho si abandonara o dejara a medias una transacción.

Este compromiso a su vez requiere que el negociador no sólo cumpla con su misión, sino que tenga la visión clara de lo que va a ocurrir si se resuelve el conflicto en la forma A, en la forma $\mathrm{B}$ o en cualquier otra forma específica, puesto que la perspectiva tiene que ver con la representación mental que efectúa el negociador anticipándose a los acontecimientos; tales posibilidades le plantean riesgos que pueden irritarlo emocionalmente, exigiendo al 
negociador el control emocional que no se consigue sólo deseándolo, sino al parecer existe como un factor inherente a la personalidad.

El presente estudio, al encontrar que estos factores se correlacionan fuertemente con la puntuación total, nos muestra que son partes constitutivas del todo medido, en la negociación del conflicto, por tanto podemos afirmar que el modelo asumido es válido y el instrumento desarrollado lo mide dentro del margen de error permitido.

\section{CONCLUSIONES}

1. El test de habilidades para la gestión en la negociación de conflictos resulta válido por los métodos de contenido y constructo.

2. El test de habilidades para la gestión en la negociación de conflictos ha demostrado confiabilidad en la medición por los métodos de la consistencia interna por la fórmula de Cronbach y por el método del test retest.

\section{REFERENCIAS BIBLIOGRÁFICAS}

1. Deutsch, M. (1993). The resolutión of conflict: Constructive and destructive processes. Yale University Press, New Haven.

2. Jenney, R.W., y Arendt, H. (1992). "Intracultural Tact vs. Intercultural Tact”, en Wats, R.J., e IDE, S. (eds). Politeness in Language. Estudies in its history, teory and practice, Mouton-De Gruyter, Berlin, pp. 21-41.

3. Malaret, J. (2001). Manual de negociación y mediación. Madrid: Colex.

4. Munduate, L., y Martínez Riquelme, J.M. (1998). Conflicto y negociación. Madrid: Pirámide,

5. Rodríguez, J.M. (1993). El factor humaño en la empresa, Deusto, Bilbao, pp.91105. 


\section{ANEXO}

\section{TEST DE H.H. L.G. EN L.N.D C. 2006}

Dr. Luis Alberto Vicuña Peri

\section{Instrucciones}

Este es un test que le permitirá a usted conocer la forma cómo aborda situaciones donde participará de manera activa en el abordaje y solución de conflictos. Para lo cual deberá contestar las preguntas que a continuación se reproducen escribiendo una " $\mathrm{x}$ " dentro de la celda que mejor describa su respuesta.

No hay respuestas buenas ni malas, sólo interesa la forma como usted siente y percibe el momento actual, de ello dependerá la validez y la confiabilidad de sus resultados.

Conteste de la forma siguiente:

- Marque el 1 si está definitivamente en desacuerdo.

- Marque el 2 si está muy en desacuerdo.

- Marque el 3 si está desacuerdo.

- Marque el 4 si está de acuerdo.

- Marque el 5 si está muy de acuerdo.

- Marque el 6 si está definitivamente de acuerdo.

\begin{tabular}{|c|c|c|c|c|c|c|c|}
\hline \multirow[b]{2}{*}{$\mathbf{N}^{\mathbf{0}}$} & \multirow[b]{2}{*}{ Pregunta } & \multicolumn{3}{|c|}{ Desacuerdo } & \multicolumn{3}{|c|}{ Acuerdo } \\
\hline & & 1 & 2 & 3 & 4 & 5 & 6 \\
\hline 1. & $\begin{array}{l}\text { Me resulta fácil aislar de una situación conflictiva el motivo } \\
\text { principal de lo secundario. }\end{array}$ & & & & & & \\
\hline 2. & Con mi postura corporal comunico mensajes. & & & & & & \\
\hline 3. & $\begin{array}{l}\text { Cuando participo en la solución de un conflicto me es fácil } \\
\text { ponerme en el punto de referencia de cada uno. }\end{array}$ & & & & & & \\
\hline 4. & $\begin{array}{l}\text { Cuando estoy en el medio de un conflicto lo vivo tan } \\
\text { intensamente aportando en su solución. }\end{array}$ & & & & & & \\
\hline 5. & $\begin{array}{l}\text { Logro comprender el estado conflictivo de mi interlocutor aún } \\
\text { cuando procede de una cultura diferente. }\end{array}$ & & & & & & \\
\hline 6. & $\begin{array}{l}\text { Cuando me hablan y ruidos impiden escuchar parte del } \\
\text { mensaje, con lo que oí basta para completarlo. }\end{array}$ & & & & & & \\
\hline 7. & $\begin{array}{l}\text { Por los gestos de mi interlocutor sé que piensa que me está } \\
\text { engañando. }\end{array}$ & & & & & & \\
\hline 8. & $\begin{array}{l}\text { Cuando una persona me cae mal y solicita mi atención, tengo } \\
\text { la fluidez para derivarla a otra persona. }\end{array}$ & & & & & & \\
\hline
\end{tabular}




\begin{tabular}{|c|c|c|c|c|c|c|c|}
\hline \multirow{2}{*}{$\mathbf{N}^{\mathbf{0}}$} & \multirow{2}{*}{ Pregunta } & \multicolumn{3}{|c|}{ Desacuerdo } & \multicolumn{3}{|c|}{ Acuerdo } \\
\hline & & 1 & 2 & 3 & 4 & 5 & 6 \\
\hline 9. & $\begin{array}{l}\text { Huyo de aquellas personas que me tratan como su "paño de } \\
\text { lagrimas" con sus problemas. }\end{array}$ & & & & & & \\
\hline 10. & Me es difícil proporcionar los datos para obtener un identikit. & & & & & & \\
\hline 11. & $\begin{array}{l}\text { Al encontrarme con una persona me es fácil que se sienta en } \\
\text { confianza. }\end{array}$ & & & & & & \\
\hline 12. & $\begin{array}{l}\text { Me es difícil ponerme en el punto de referencia de dos } \\
\text { personas en conflicto, generalmente me inclino por una. }\end{array}$ & & & & & & \\
\hline 13. & $\begin{array}{l}\text { Cuando participo en la solución del conflicto, más que por } \\
\text { vocación, lo hago por que me siento obligado. }\end{array}$ & & & & & & \\
\hline 14. & $\begin{array}{l}\text { Cuando vivo un problema hago hasta lo imposible con el } \\
\text { propósito que solo quede para mí. }\end{array}$ & & & & & & \\
\hline 15. & $\begin{array}{l}\text { Cuando voy a una tienda y veo una vitrina me resulta fácil } \\
\text { imaginarme las partes que no veo. }\end{array}$ & & & & & & \\
\hline 16. & Me es fácil comunicar lo que siento con gestos. & & & & & & \\
\hline 17. & $\begin{array}{l}\text { La expresión de mi rostro logra que los gestos molestosos de } \\
\text { mi interlocutor se atenúen. }\end{array}$ & & & & & & \\
\hline 18. & $\begin{array}{l}\text { Me cuesta conectarme con el tema conflictivo, permanece en } \\
\text { mí lo que debe ser desde mi punto de vista. }\end{array}$ & & & & & & \\
\hline 19. & $\begin{array}{l}\text { Cuando escribo un dictamen o notas según el mensaje, imagino } \\
\text { la cara que pondrá el lector. }\end{array}$ & & & & & & \\
\hline 20. & $\begin{array}{l}\text { Me es difícil ponerme en el punto de referencia del conflicto de } \\
\text { personas de una comunidad campesina serrana. }\end{array}$ & & & & & & \\
\hline 21. & $\begin{array}{l}\text { Me resulta fácil encontrar en las personas el lado agradable que } \\
\text { elimine mi fastidio emocional. }\end{array}$ & & & & & & \\
\hline 22. & $\begin{array}{l}\text { Tengo la habilidad para descifrar los mensajes ambiguos sin } \\
\text { preguntar a mi interlocutor. }\end{array}$ & & & & & & \\
\hline 23. & $\begin{array}{l}\text { Me entretengo buscando la forma como lograr que las personas } \\
\text { metidas en un lío lo resuelvan satisfactoriamente. }\end{array}$ & & & & & & \\
\hline 24. & $\begin{array}{l}\text { Según mi plasticidad para ponerme en el punto de referencia de } \\
\text { las personas en conflicto gestiono la solución. }\end{array}$ & & & & & & \\
\hline 25. & $\begin{array}{l}\text { Me agrada que mi interlocutor se exprese con claridad y } \\
\text { brevedad. }\end{array}$ & & & & & & \\
\hline 26. & $\begin{array}{l}\text { Logro que los demás me reconozcan demostrándoles mi interés } \\
\text { por ayudarles a resolver sus problemas. }\end{array}$ & & & & & & \\
\hline 27. & $\begin{array}{l}\text { Resuelvo con facilidad la posición en que quedará una moneda } \\
\text { cuando al caer ha girado } 10 \text { veces. }\end{array}$ & & & & & & \\
\hline 28. & $\begin{array}{l}\text { Me resulta difícil ponerme en el punto de referencia del } \\
\text { conflicto de funcionarios corruptos. }\end{array}$ & & & & & & \\
\hline 29. & Tengo la habilidad para anticiparme a lo que me van a decir. & & & & & & \\
\hline 30. & $\begin{array}{l}\text { Tengo la habilidad para lograr en situaciones de conflicto que } \\
\text { las personas regulen o controlen su estrés. }\end{array}$ & & & & & & \\
\hline 31. & $\begin{array}{l}\text { Tengo buen olfato para detectar problemas y alejarme antes } \\
\text { que revienten. }\end{array}$ & & & & & & \\
\hline 32. & $\begin{array}{l}\text { Vivo intensamente el estado emocional de mi interlocutor aún } \\
\text { cuando personalmente es de mi desagrado. }\end{array}$ & & & & & & \\
\hline
\end{tabular}




\begin{tabular}{|c|c|c|c|c|c|c|c|}
\hline \multirow[b]{2}{*}{$\mathbf{N}^{\mathbf{0}}$} & \multirow[b]{2}{*}{ Pregunta } & \multicolumn{3}{|c|}{ Desacuerdo } & \multicolumn{3}{|c|}{ Acuerdo } \\
\hline & & 1 & 2 & 3 & 4 & 5 & 6 \\
\hline 33. & $\begin{array}{l}\text { Siento la barrera sociocultural cada vez que me comunico con } \\
\text { extraños. }\end{array}$ & & & & & & \\
\hline 34. & $\begin{array}{l}\text { Me cuesta imaginarme cómo me veré en una foto vestido } \\
\text { estrafalariamente. }\end{array}$ & & & & & & \\
\hline 35 . & $\begin{array}{l}\text { Si me ofrecen un trabajo como conciliador lo acepto a menos } \\
\text { que tenga otra oferta mejor. }\end{array}$ & & & & & & \\
\hline 36. & $\begin{array}{l}\text { Me cuesta mucho lograr que las personas me perciban como lo } \\
\text { que soy emocionalmente, sosegado, tranquilo, etc. }\end{array}$ & & & & & & \\
\hline 37. & Entiendo el conflicto desde el punto de vista del interlocutor. & & & & & & \\
\hline 38. & $\begin{array}{l}\text { Tengo la disposición de meterme de lleno en los problemas de } \\
\text { otros contribuyendo a la solución. }\end{array}$ & & & & & & \\
\hline 39. & Soporto la mirada fija de mi interlocutor atenuándolo. & & & & & & \\
\hline 40. & $\begin{array}{l}\text { Tengo el don, según me dicen, de calmar a las personas con } \\
\text { tan solo mi presencia. }\end{array}$ & & & & & & \\
\hline 41. & $\begin{array}{l}\text { Sé como apartarme de líos ajenos sin que estos se den por } \\
\text { ofendidos. }\end{array}$ & & & & & & \\
\hline 42. & Vivo un relato como si estuviese presente en él. & & & & & & \\
\hline 43. & $\begin{array}{l}\text { Las propuestas de solución emanan tener en cuenta, como se } \\
\text { sentirán las personas al resolver el conflicto. }\end{array}$ & & & & & & \\
\hline 44. & $\begin{array}{l}\text { Me resulta fácil lograr en cualquier situación que las personas } \\
\text { muestren su lado agradable. }\end{array}$ & & & & & & \\
\hline 45. & Antes de preguntar escucho completamente a mi interlocutor. & & & & & & \\
\hline 46. & $\begin{array}{l}\text { Por humanidad colaboro en la solución de conflictos a costa de } \\
\text { mi tranquilidad. }\end{array}$ & & & & & & \\
\hline 47. & $\begin{array}{l}\text { Cuando ingreso a edificios de diferentes niveles al salir } \\
\text { comúnmente me desoriento. }\end{array}$ & & & & & & \\
\hline 48. & $\begin{array}{l}\text { Controlo el estrés en situaciones de conflicto de forma tal que } \\
\text { termino agotado. }\end{array}$ & & & & & & \\
\hline 49. & $\begin{array}{l}\text { Me es difícil ponerme en el punto de referencia del conflicto de } \\
\text { una persona de la amazonía. }\end{array}$ & & & & & & \\
\hline 50 . & $\begin{array}{l}\text { Me resulta difícil identificar a una persona conocida al verla } \\
\text { después de diez años. }\end{array}$ & & & & & & \\
\hline
\end{tabular}

Gracias

Ahora espere instrucciones del examinador 